Musées, Patrimoine et Culture scientifiques et techniques

$194 \mid 2021$

mars-avril 2021

\title{
Faire entrer le corps et l'attention au musée
}

\section{Véronique Antoine-Andersen}

\section{OpenEdition \\ Journals}

Édition électronique

URL : https://journals.openedition.org/ocim/4234

DOI : $10.4000 /$ ocim.4234

ISSN : 2108-646X

Éditeur

OCIM

Édition imprimée

Date de publication : 1 mars 2021

Pagination : 18-23

ISSN : 0994-1908

Référence électronique

Véronique Antoine-Andersen, "Faire entrer le corps et l'attention au musée », La Lettre de l'OCIM [En

ligne], 194 | 2021, mis en ligne le 01 mars 2021, consulté le 11 avril 2022. URL : http://

journals.openedition.org/ocim/4234; DOI : https://doi.org/10.4000/ocim.4234

Ce document a été généré automatiquement le 11 avril 2022

Tous droits réservés 


\title{
Faire entrer le corps et l'attention au musée
}

\author{
Véronique Antoine-Andersen
}

Visite sensorielle au Frac de Franche-Comté.

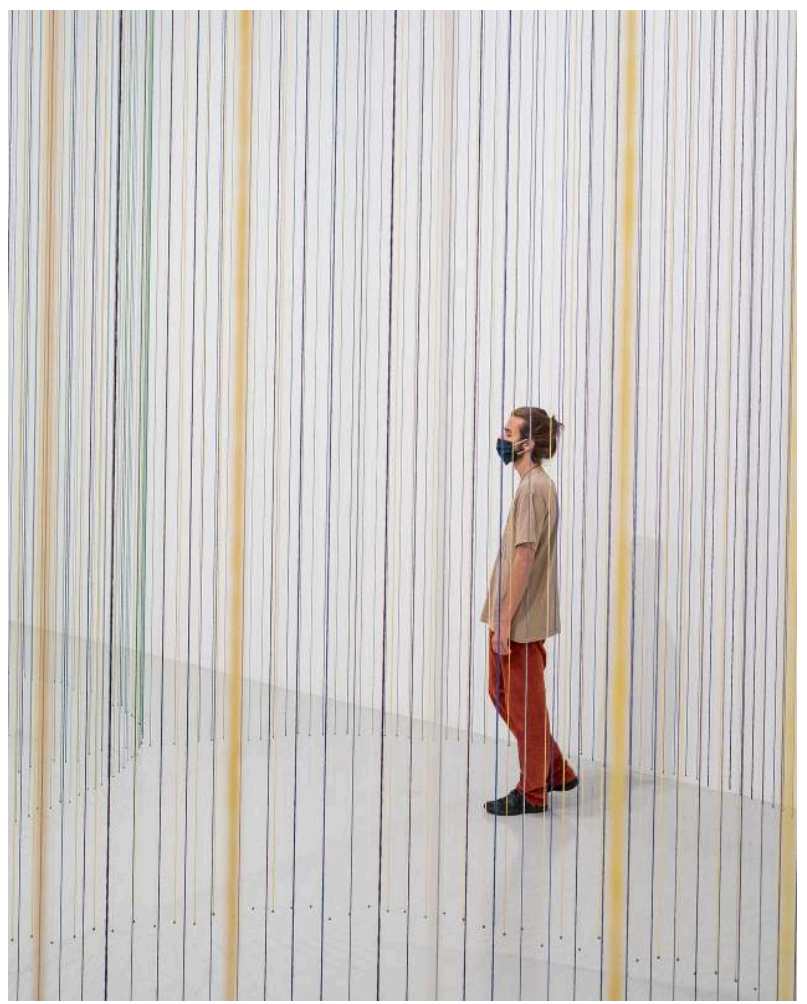

(c) Frac Franche-Comté, 2020. Photo : Nicolas Waltefaugle

1 La crise écologique et environnementale a révélé une génération de philosophes et de scientifiques qui repensent notre rapport au vivant et renouvellent notre manière de penser le monde. Parmi ces intellectuels de terrain, Baptiste Morizot, enseignant 
chercheur en philosophie, naturaliste, maitre de conférence à l'université d'AixMarseille pratique le pistage et s'intéresse tout particulièrement aux relations entre le genre humain et le vivant. Selon lui, la crise écologique constitue une crise de la sensibilité - une crise de la sensibilité envers le monde vivant. Par crise de la sensibilité, il entend un appauvrissement de ce que nous pouvons sentir, percevoir, comprendre, et tisser comme relations à l'égard du vivant. Une réduction de la gamme des affects, des percepts, et des concepts nous reliant à lui.

\section{Une crise de la sensibilité aux œuvres d'art}

2 Cet appauvrissement de la sensibilité au vivant fait écho, même si elle ne lui est pas apparentée, à la crise de la sensibilité aux œuvres d'art. Pour s'en convaincre, il suffit de se poster dans une salle d'exposition et observer les modes opératoires des visiteurs. L'appauvrissement relationnel vis-à-vis des œuvres d'art s'exprime à travers un indicateur fiable, celui de la durée passée devant une œuvre d'art. Une enquête quantitative de 1999, menée par Jean-Claude Passeron et Emmanuel Pedler intitulée Le temps donné au regard. Enquête sur la réception de la peinture ${ }^{1}$ privilégiait parmi d'autres indicateurs, le temps de visionnement consacré par les visiteurs à une trentaine d'œuvres présentées dans deux salles du musée Granet à Aix-en-Provence. Sachant que l'expérience de l'art est une chose complexe à décrire, l'enquête soulignait que cet indicateur fournissait un indice significatif quant à l'intensité interne de l'expérience vécue et révélait indéniablement le degré d'implication et d'engagement dans la relation à l'œuvre. Les chiffres concernant cet indicateur sont parlants. En 2008, le philosophe Bernard Stiegler, dans un colloque au Louvre, évoquait une durée moyenne de 40 secondes passées devant les œuvres du Louvre. Cette durée aujourd'hui est en baisse dans l'ensemble des musées et s'établit à une dizaine de secondes. La Tate Gallery parle de huit secondes.

3 Dans un laps de temps si court, l'œil est-il en mesure d'apprivoiser une œuvre ? Même si le contact avec l'art provoque occasionnellement des émotions chocs, assimilables au coup de foudre amoureux, le reste du temps, une dizaine de secondes s'avère insuffisante pour prétendre entrer en contact avec une œuvre, la parcourir dans son entier, se laisser traverser par elle afin qu'elle puisse œuvrer sur nous. Incontestablement, toute rencontre s'établit dans la durée. Ce regard éclair relève d'une forme de cécité qui ne dit pas son nom et nous fait passer à côté de l'œuvre, au sens propre et figuré. Pour les professionnels de musées que nous sommes, cette donnée significative mérite qu'on s'y arrête, qu'on l'interroge, qu'on identifie ses causes afin d'être en mesure d'inventer de nouveaux modes opératoires aptes à revivifier, redynamiser la relation des publics vis-à-vis des œuvres d'art. Les chiffres élevés de fréquentation des grandes institutions parisiennes entre 2018 et 2019 ne contredisent pas cette réalité. 


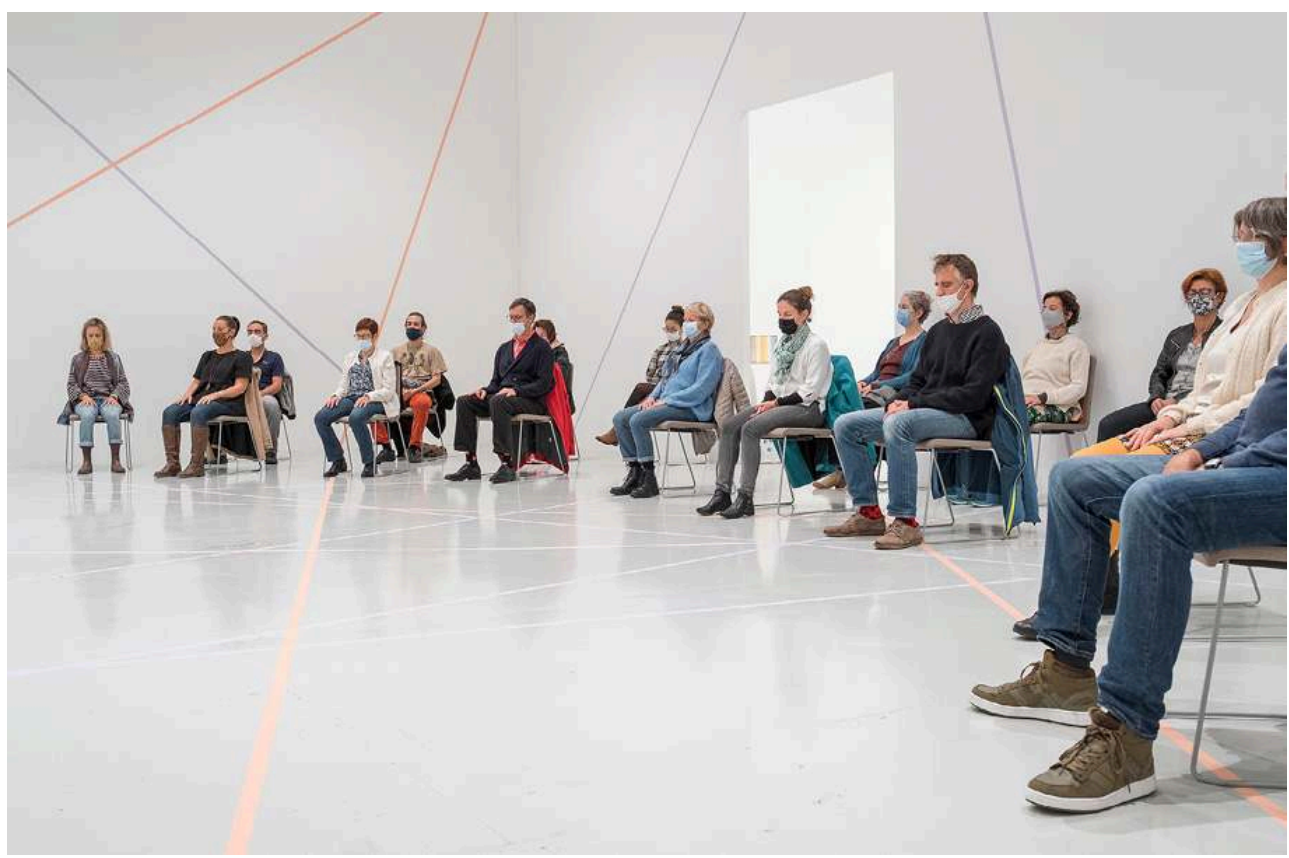

(c) Frac Franche-Comté, 2020. Photo : Nicolas Waltefaugle

\section{La visite, une expérience sensorielle}

La bataille culturelle vis-à-vis du vivant défendue par Baptiste Morizot et des ouvrages, comme celui du scientifique Jacques Tassin paru en 2019, Pour une écologie du vivant, peuvent nous ouvrir des pistes. Elle consiste en un enrichissement et un pluralisme des relations possibles avec le vivant mais aussi à une réanimation des savoirs, au sens premier du terme c'est-à-dire, redonner du souffle à ces savoirs en les mettant au contact d'une expérience sensorielle du vivant.

Il ajoute qu'une culture artistique n'est pas essentiellement une accumulation de savoirs et qu'elle mobilise des types d'attention spécifiques selon les objets observés et dont la transmission s'est aujourd'hui perdue. C'est uniquement au contact du vivant que ces formes d'attention peuvent se redéployer et, ce faisant, revivifier notre sensibilité au vivant. De la même façon, l'art de regarder une œuvre d'art est un phénomène perceptif qui mobilise à la fois la sensation, le cognitif, les affects, la mémoire, les expériences vécues et aussi une forme d'attention spécifique que JeanMarie Schaeffer qualifie d'attention esthétique. Il serait temps que les futurs médiateurs acquièrent lors de leurs études, les bases élémentaires de la perception afin de déjouer des idées fausses à ce sujet, à commencer par la plus commune, que nous voyons tous la même chose. Cette méconnaissance de la part des personnes en charge de la médiation est dommageable, il me semble, parce qu'elle réduit le champ des possibles en ce qui concerne la création de nouveaux formats de visite.

Toute activité culturelle engage une somme de gestes et de manières d'utiliser son corps qui constituent une technique du corps, selon la définition de Marcel Mauss, afin de favoriser la réception de l'œuvre. En ce qui concerne le musée, on remarque que les visiteurs, passé le seuil des salles, épousent une série de gestes spécifiques comme ralentir le pas, baisser le ton de la voix, mettre les bras au repos en les croisant devant 
ou derrière leur dos, adapter sa démarche à celle des visiteurs environnants... Cette somme de gestes transmise par mimétisme ne fait pas l'objet d'un apprentissage spécifique dans les formats de visite proposés, pour la simple raison que la médiation culturelle s'intéresse peu à cet aspect physico-perceptif du visiteur considérant qu'il n'est pas essentiel dans la sensibilisation aux œuvres d'art.

\section{Le corps du visiteur}

7 Or, il me semble que justement, prendre en considération le corps du visiteur dans son entier et cultiver ses capacités d'attention constituent des leviers disponibles et efficaces pour revivifier la relation à l'art des visiteurs. Dans un ouvrage ${ }^{2}, \mathrm{j}^{\prime}$ ai imaginé un protocole intitulé la Cérémonie du Regard qui s'adresse à tous les visiteurs individuels, désireux d'approfondir et d'élargir leur relation à l'art. Placé sous le signe du ralentissement, ce protocole attentionnel et visuel engage le corps, l'intuition, le sensible, l'attention et l'intelligence du visiteur de manière à lui faire éprouver l'œuvre et vivre à son contact, une expérience globale ou holistique. Il s'agit d'une piste majeure à débroussailler aujourd'hui par les professionnels de la médiation culturelle.

8 Enseignant et théoricien de la couleur, Johannes Itten avait l'habitude lorsqu'il dirigeait le cours préliminaire de l'école du Bauhaus, de commencer ses cours par une relaxation du corps, considérant qu'un corps tendu n'était pas disposé à la création. L'état physique influe sur la réceptivité artistique et visiter une exposition dans un corps mal en point peut vite tourner au cauchemar. Si le corps et l'esprit sont deux objets distincts, l'homme n'est pas un corps et un esprit, mais un corps avec un esprit, les deux étant étroitement connectés et impliqués dans la perception esthétique. Le rôle du corps ne se réduit pas à un moyen de locomotion. Cette prise en considération du corps est une réalité, trop souvent négligée par les professionnels des musées, qui n'hésitent pas, pour des raisons de circulation et de sécurité, à supprimer les sièges dans les espaces d'exposition.

9 Le visiteur considéré comme un être désincarné ? Alors que les sciences cognitives, la phénoménologie et les neurosciences montrent que le corps était un récepteur actif, contribuant pleinement à notre compréhension du monde et de nos existences, encore aujourd'hui, le monde occidental et plus particulièrement, le pays de Descartes, continuent d'adhérer à cette suprématie de l'esprit et du concept. La médiation culturelle, largement relayée par la connaissance et le savoir, n'échappe pas à cette emprise. Le visiteur est d'abord un cerveau avant d'être un corps, un être de raison plutôt qu'un être "incorporé " ou unifié. Or c'est par nos sens que nous entrons en contact avec le monde et l'art ; c'est eux qui nous fournissent une image de ce qui nous entoure. Le corps humain est une entité savante, il est la structure, le producteur et le conservateur du savoir existentiel. Le savoir n'est pas seulement moulé dans des mots, concepts et théories. Lorsqu'elle sollicite principalement la raison au détriment des sens, la visite guidée escamote cette réalité et atrophie le contact global du visiteur avec les œuvres.

10 L'observateur fait partie de l'observation écrivait Marcel Mauss. Des musées à Lille, Lyon et bien d'autres villes encore, l'ont bien sûr déjà compris en offrant des expériences de visite innovantes où le corps et les sens reprennent leur place. Je citerai l'exemple d'une visite au musée Guimet où nous étions invités à exécuter des postures de yoga pour être mieux disposés à voir des statues indiennes. Ces alternatives 
participent du renouvellement indispensable du musée, compte tenu des grands bouleversements qui l'affectent, telles la mondialisation, la diversité élargie des publics et de leurs attentes, la révolution numérique, la crise de l'attention, la mutation des outils de transmission des savoirs... Le colloque Vie des musées, Temps des publics ${ }^{3}$ organisé par le ministère de la Culture proposait d'ailleurs des ateliers à ce sujet.

\section{Slow visite, une nouvelle forme de médiation} méditation au coeur de l'exposition plonge le visiteur dans une nouvelle expérience de réception et de dialogue avec l'art. Ce temps de méditation est suivi d'une "slow visite". Toucher les cuvres avec nos yeux, au-delà de ce que nos yeux perçoivent... telle est l'intention des formatrices, Natalie Grosjean et Marie-Pierre Le Bris, qui accompagnent les visiteurs dans cette découverte différente des expositions, révélant ainsi les cuvres dans toute leur dimension sensible».

年 avec l'art ", " découverte différente » informe sur les aspects innovants et expérimentaux de cette programmation. Par ailleurs, le mot "méditation » est employé sans équivoque indiquant clairement l'orientation et le ton de cette visite. L'appellation slow visite réfère au Slow looking. Cette expression employée dans les pays anglo-saxons définit une manière de découvrir une œuvre d'art en lui accordant du temps afin de pouvoir s'en imprégner et créer une relation intime avec elle. Le critique d'art américain Peter Clothier a écrit en 2013, un ouvrage intitulé Slow Looking ${ }^{4}$, un guide du slow looking ${ }^{5}$ est disponible sur le site de la Tate Gallery de Londres ainsi que des visites intitulées Looking without talking. Depuis 2013 également, aux États-Unis, l'Américain Phil Terry a initié un rendez-vous annuel, début avril, dans les musées volontaires, qui s'intitule le Slow Art Day et dont la mission est d'apprendre au plus grand nombre de visiteurs à regarder et à aimer l'art. Cette journée programmée désormais dans de nombreux musées et galeries anglo-saxons, commence à gagner du terrain en Europe, notamment aux Pays-Bas, en Irlande et en Belgique. Cette initiative reste totalement méconnue en France.

\section{La pleine conscience comme préambule à la visite}

Revenons à la visite. Une fois le groupe rassemblé dans l'entrée du Frac, la directrice du Frac commence par présenter très rapidement l'exposition temporaire dédiée à Cécile Bart et l'expérience d'immersion dans cette œuvre proposée par Natalie Grosjean, psychologue et animatrice de formations de pleine conscience. Puis Natalie Grosjean se présente et nous enjoint de la suivre pour nous rendre à la salle d'exposition, avec comme consigne, lorsque nous monterons l'escalier, de tenter d'être présent à $100 \%$ à ce qui se présente devant nos yeux ainsi qu'au contact des sensations de nos pieds en train de monter les marches. Dans la première salle de l'exposition, des chaises ont été 
installées face à une œuvre sur lesquelles nous sommes invités à prendre place. Covid oblige, nous sommes masqués et installés sur des chaises distancées. Natalie Grosjean nous invite d'abord à nous asseoir confortablement et à ressentir le contact physique de notre corps sur la chaise, ainsi que celui de nos pieds sur le sol. Puis, elle introduit en quelques mots la pleine conscience avant de nous donner le mode d'emploi de la visite : regarder en essayant d'être pleinement présent aux œuvres présentées dans les salles, ne pas laisser l'esprit vagabonder vers nos activités passées de la journée ou nos projets futurs, varier les points de vue, ralentir sa démarche, aller au grés de nos envies, avancer et reculer, s'approcher pour mieux percevoir un détail, reculer encore, reposer le regard en le laissant s'évader par la fenêtre, s'arrêter, fermer les yeux. Voici quelques exemples des préconisations énoncées. Puis, pendant quelques minutes, elle nous propose de porter notre attention sur notre respiration, en fermant ou non, les yeux.

\section{La visite avec l'attention à l'exposition}

L'introduction a duré une vingtaine de minutes. Ensuite, chaque visiteur entame sa pérégrination dans les trois salles de l'étage. Dans cette introduction, aucune mention n'a été faite de l'art en général, ni de l'artiste, en particulier. Nous partons avec pour seul guide, une sorte de mode d'emploi comportemental qui intègre le corps et l'esprit. Pendant quarante-cinq minutes, la vingtaine de visiteurs déambule dans les salles adoptant une démarche naturellement ralentie, opérant des déplacements autour des œuvres rythmés par des pauses plus longues qu'à l'accoutumée. La tête des visiteurs est sollicitée plus activement, engagée dans des positions variées pour entrevoir l'œuvre dans ses différents états. Certains cheminent puis marquent une pause pour revoir de loin, les œuvres approchées préalablement.

Corps en mouvement durant une visite pleine d'attention.

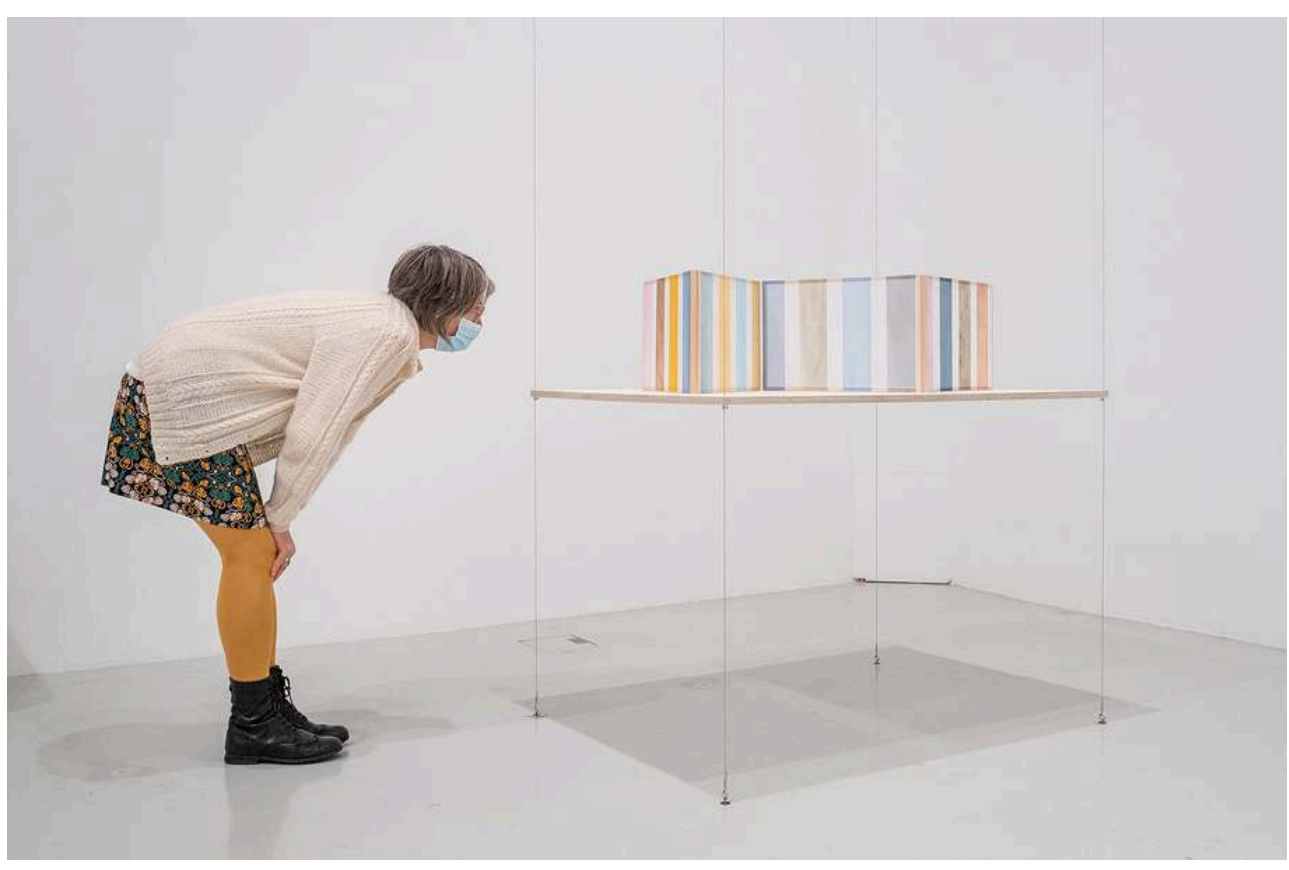

(c) Frac Franche-Comté, 2020. Photo : Nicolas Waltefaugle 
17 Des postures observables, me direz-vous, dans toutes les salles d'exposition ? Non, parce que le discours d'introduction malgré sa brièveté conditionne de façon très spectaculaire, les comportements de tous les visiteurs. C'est palpable, sensible et les photos prises lors de la séance ne peuvent pas en rendre compte. Ce qui se joue là est de l'ordre du ressenti, du sensible. Je me surprends à adopter naturellement une cadence ralentie, je ressens des sensations tactiles fines au contact de la matière papier des œuvres, j'éprouve physiquement les couleurs vives et saturées. Je sens mon corps particulièrement mobilisé et réactif. Le ralentissement de nos déplacements corporels conditionne une autre manière de nous relier à l'œuvre. Il impose un rythme qui stimule la curiosité, la rêverie, cette capacité selon Gaston Bachelard à investir l'instant. Des références artistiques, des images viennent se juxtaposer, enrichir l'investigation visuelle et nourrir l'invention d'un récit personnel autour de ces œuvres sur lesquelles nous n'avons reçu aucune information. Je m'autorise davantage de retours en arrière. Le temps semble suspendu. L'expérience est surprenante, la vieille routarde des musées que je suis, en convient.

\section{Partage de ressentis, vécus des visiteurs}

Quarante-cinq minutes se déroulent avant de se rassembler pour descendre dans la dernière salle de l'exposition qui présente une installation sonore avec des films projetés au mur et au sol. Natalie Grosjean, sensible au corps du visiteur, nous invite à nous asseoir si nous en ressentons le besoin, avant de pénétrer dans cette ultime salle. Pour finir, un échange d'expériences est proposé lors duquel trois visiteurs expriment leur surprise de l'impact du " conditionnement " reçu au départ sur la qualité de leur visite. Ils constatent une augmentation de l'acuité de leur regard et la satisfaction procurée en retour. Ils en profitent pour exprimer leurs ressentis sur les œuvres. Il est intéressant de noter que personne ne s'est plaint de l'absence d'informations sur l'œuvre de Cécile Bart. Ce "conditionnement ", mot employé par les visiteurs, s'apparente au final à une technique du corps proposée par Natalie Grosjean centrée sur le ralentissement, l'instantanéité, et le redéploiement de l'attention qui engendre l'ouverture, la disponibilité.

Cette expérience innovante a tenu ses promesses indéniablement et semble parfaitement convenir à un certain type de public. Elle fournit une matrice, un matériau à partir duquel pourrait s'élaborer d'autres formats de visites hybrides qui mêleraient, à la fois, technique du corps et discours sur l'œuvre. Pour faire émerger des offres alternatives au sein des musées, aller se frotter à d'autres disciplines, comme les neurosciences, l'anthropologie, les sciences du vivant, la philosophie... ouvre des horizons passionnants et prometteurs. Il nous revient de nous en saisir. 


\section{NOTES}

1. Passeron J.-C. et Pedler E. Le temps donné au regard. Enquête sur la réception de la peinture, Protée, vol. 27, n², 1999, pp. 93-116 : https://doi.org/10.7202/030563ar

2. Antoine-Andersen V. Regarder l'art et aimer ça. Paris : Eyrolles, 2019, 110 p.

3. Colloque organisé par le ministère de la Culture du 21 au 23 juin 2017.

4. Clothier P. Slow Looking. Toad Rampant Publications, 2012.

5. https://www.tate.org.uk/art/guide-slow-looking

\section{RÉSUMÉS}

De nombreuses mutations observées ces dernières années comme la révolution numérique ou la crise de l'attention impactent profondément le visiteur de musée dans son rapport aux œuvres. Prenant en compte ces nouveaux paradigmes, l'autrice en appelle au renouvellement de la médiation culturelle, tant au niveau de ses propositions que de sa formation.

\section{INDEX}

Mots-clés : métier/personnel de la médiation, médiation

\section{AUTEUR}

\section{VÉRONIQUE ANTOINE-ANDERSEN}

A travaillé successivement au musée en Herbe, au Centre Pompidou, à la Halle saint Pierre et à la Cité de l'architecture où elle a rempli différentes missions. Autrice du livre Regarder une cuvre d'art et aimer ça.

vu.andersen@free.fr 